\section{APORTACIÓN DE LA MUJER MILITAR EN LAS FUERZAS ARMADAS ESPAÑOLAS A LA DEFENSA Y LA SEGURIDAD}

\author{
María Belén Caballud Hernando \\ Vocal del observatorio militar de igualdad \\ Ministerio de Defensa de España \\ belencab@oc.mde.es
}

Cómo citar este artículo/Citation: Caballud Hernando, M. B. (2014). "Aportación de la mujer militar en las Fuerzas Armadas españolas a la Defensa y la Seguridad". Arbor, 190 (765): a099. doi: http://dx.doi.org/10.3989/arbor.2014.765n1006

Recibido: 5 junio 2012. Aceptado: 2 diciembre 2013.

RESUMEN: La contribución de las mujeres de forma activa y profesional a la Defensa y Seguridad de nuestro país se ha consolidado como una cuestión de derecho, de eficacia, eficiencia y operatividad, redundando en la seguridad de todos. Han trascurridos 25 años desde que se abrieron las puertas a la incorporación a la mujer a las FAS. El proceso normativo ha sido gradual, tardío en el tiempo con respecto a los países de nuestro entorno pero rápido en su evolución. Hoy día la mujer militar española puede acceder a cualquier destino profesional existente en las FAS. Sin embargo todavía hay que seguir trabajando para que la igualdad efectiva entre mujeres y hombres militares sea una realidad completa.

PALABRAS CLAVE: militar; defensa; conciliación; mujer; género.

\section{CONTRIBUTION OF MILITARY WOMEN IN THE SPANISH ARMED FORCES TO DEFENCE AND SECURITY}

Copyright: (C) 2014 CSIC. Este es un artículo de acceso abierto distribuido bajo los términos de la licencia Creative Commons Attribution-Non Commercial (by-nc) Spain 3.0.

ABSTRACT: Women's contribution to national defence and security has become established as a matter of law, effectiveness, efficiency and operability, benefiting the security of all. Twenty-five years have passed since the armed forces opened their doors to women. The regulatory process has been gradual and late compared to neighbouring countries, but has evolved rapidly. Today, Spanish military women can access any existing professional destination in the armed forces. However, there is still a long way to go to achieve effective equality between women and men in the Armed Forces.

KEYWORDS: military; defence; conciliation; women; gender. 
En 25 años las Fuerzas Armadas españolas han sido protagonistas de una profunda transformación y cambio que ha afectado tanto a su organización y misiones, como a su reclutamiento y composición.

Hasta la última década del siglo XX, nuestros Ejércitos contaban con personal voluntario y forzoso, procedente del servicio militar obligatorio, exclusivamente masculino, y con unas funciones de Defensa más concretas o delimitadas objetiva y subjetivamente ${ }^{1}$. En la actualidad, España cuenta con unas Fuerzas Armadas profesionales, compuestas e integradas por hombres y mujeres voluntarias, y con una importante proyección internacional en cuanto a actuaciones y responsabilidades ${ }^{2}$.

Entre los elementos fundamentales del cambio destacan la incorporación de España a Organizaciones Internacionales de Seguridad y Defensa y la presencia de mujeres y hombres en nuestras Fuerzas Armadas. La evolución de esta última realidad ha ido, como veremos, paralela a la profesionalización de las mismas.

\section{LA MUJER EN LAS FUERZAS ARMADAS ESPAÑOLAS}

La incorporación de la mujer en las Fuerzas Armadas españolas es una conquista muy reciente, de finales del siglo XX. Se produce 10 años después de la promulgación de nuestra Carta Magna, la Constitución de 1978, que consagra la igualdad entre mujeres y hombres y la no discriminación por razón de sexo, asimismo, en su artículo 30 establece el derecho y el deber de todos los españoles de defender a España. La puesta en práctica de este principio constitucional de igualdad en este ámbito castrense, no fue inmediato.

En $1988^{3}$, mediante un Real Decreto-Ley, las Fuerzas Armadas españolas abrieron sus puertas a la incorporación de mujeres entre sus filas, si bien, de una manera muy limitada, y sólo a determinados Cuerpos, Escalas y Categorías. Ese primer paso fue dado por veinticinco mujeres que desafiaron a quienes opinaban que la profesión militar no era un ámbito propio de mujeres, rompieron, sin duda, estereotipos de género normales hasta entonces. Ingresaron a los hoy denominados Cuerpos Comunes y a las Ingenierías de los Ejércitos y la Armada. En ese momento se produjo una ruptura de la dicotomía histórica en cuanto a las profesiones masculinas y femeninas en nuestro país. Las armas y las funciones del combate no han sido tradicionalmente un ámbito propio para las mujeres.

Este proceso ha avanzado y sigue avanzado de una manera importante, aunque iniciado con un cierto retraso, al de la incorporación de la mujer a otros ámbi- tos laborales en nuestro país y respecto a la incorporación de las mujeres a otros Ejércitos.

La incorporación e integración de la mujer a las Fuerzas Armadas españolas, ha sido definido como un proceso tardío en el tiempo, respecto a otros países occidentales cuyo hecho determinante fue la Segunda Guerra Mundial, pero, por el contrario, el ritmo de ejecución ha sido notoriamente más rápido que en los países de nuestro entorno.

Este proceso se ha llevado a cabo de una manera clara, progresiva en el tiempo y escalonado, en un escenario temporal consecuente con las necesidades de nuestras Fuerzas Armadas y con la evolución de la sociedad y el marco jurídico. El proceso se ha desarrollado, en definitiva, sin prisa, pero sin pausa, estableciendo un calendario capaz de satisfacer las legítimas demandas de plena integración de las mujeres españolas y que al mismo tiempo permitiese evaluar las acciones realizadas. Podemos hablar, por tanto, de un modelo español de incorporación de la mujer, un modelo que está caracterizado por la consecución, en un primer momento, de la igualdad legal para evolucionar, una vez conseguida ésta, hacía la igualdad efectiva y real de los miembros de las Fuerzas Armadas españolas.

El modelo español de integración de las mujeres en las Fuerzas Armadas continuó en $1992^{4}$, con la aprobación del Reglamento de Tropa y Marinería Profesionales en las Fuerzas Armadas. En él se establecía que las soldados profesionales podrían optar a todos los destinos de su empleo militar, excepto los de tipo táctico $u$ operativo en determinadas unidades (como la Legión, operaciones especiales, paracaidistas y cazadores paracaidistas por razones propias de sus condiciones físicas y biológicas). Se señalaba que la mujer no podía formar parte de las fuerzas de desembarco, de las dotaciones de submarinos ni de buques menores en los que sus condiciones de habitabilidad no permiten el alojamiento en condiciones adecuadas.

Posteriormente en $1999^{5}$, la Ley de Régimen del Personal de las Fuerzas Armadas, suprime definitivamente las limitaciones para incorporarse a cualquiera de los destinos profesionales existentes en las Fuerzas Armadas. Este momento constituye la clave del proceso iniciado en 1988, en tanto que se consigue la plasmación jurídica de la igualdad formal entre las mujeres y los hombres en las Fuerzas Armadas, este hecho es jurídicamente muy importante. Esta misma norma suspende el Servicio militar obligatorio y establece las bases del modelo profesional. Desde ese 
momento los esfuerzos, al igual que en el conjunto de la sociedad civil, se centran en la consecución de la igualdad material, real y efectiva.

En ese sentido se dieron pasos como los Acuerdos de Consejo de Ministros de marzo en 2005 y 2006 en los que se adoptaron una serie de medidas de acción positiva para la integración y permanencia de la mujer en las Fuerza Armadas españolas como la creación del Observatorio de la mujer en las FAS, hoy, Observatorio Militar para la igualdad entre mujeres y hombres en las Fuerzas Armadas.

Por último, cabe citar la Ley de Carrera Militar y la Ley Orgánica de Derechos y Deberes de los miembros de las Fuerzas Armadas.

La vigente Ley de Carrera Militar ${ }^{6}$, introdujo la perspectiva de género como un principio transversal de la regulación de personal en las Fuerzas Armadas. Esta norma contiene acciones positivas relacionadas con las situaciones derivadas de la maternidad al protegerlas en diferentes momentos de la carrera militar (ingreso, ascenso, situaciones, enseñanza de formación y perfeccionamiento, destinos), regula la conciliación de la vida profesional, personal y familiar de los miembros de las Fuerzas Armadas, y asegura la representación de la mujer militar en los órganos de evaluación para la selección, ascenso y asignación de destinos.

Por su parte, la Ley Orgánica 9/2011, de 27 de julio, de derechos y deberes de los miembros de las Fuerzas Armadas reconoce expresamente el principio de igualdad y no discriminación por razón de sexo, u orientación sexual, así como el deber de promover las medidas necesarias para garantizar que, la igualdad entre el hombre y la mujer sea real y efectiva especialmente en el acceso, la prestación del servicio, la formación y la carrera militar.

De conformidad con la normativa apuntada, en nuestro país, a diferencia de los patrones seguidos por otros países, las mujeres militares actualmente pueden acceder a todos los Cuerpos y Escalas (no se han creado Cuerpos específicos para la mujeres), a todos los destinos (incluidos los más operativos y directamente relacionados con el combate) y no existen cuotas máximas para ingreso, incorporándose al mismo sistema de carrera que sus compañeros, lo que les permite poder acceder a todos los empleos.

Este marco normativo, su aplicación y el número de mujeres que ya forman parte de las Fuerzas Armadas españolas, inducen a pensar que la incorporación de la mujer a las FAS y su integración en su estructura, también operativa, es una realidad. Sin embargo no ha debido ser fácil; ha sido fruto del esfuerzo de todos, hombres y mujeres, que integran las Fuerzas Armadas.

En este sentido, se considera importante señalar como datos relevantes que, según información facilitada por el Observatorio Militar de Igualdad, en diciembre de 2012 , más del $12 \%$ del total de efectivos de las Fuerzas Armadas españolas. Son mujeres, el 6\% de los oficiales y el $17 \%$ de la tropa y marinería. El máximo empleo alcanzado por una mujer en la carrera militar es el de teniente coronel. También, es necesario subrayar, como indicador respecto a la igualdad de género en las Fuerzas Armadas, que el 13\% de los destinos de la Fuerza son ocupados por mujeres.

Cabe destacar, igualmente, que en la reciente desarrollada modalidad de incorporación a las Fuerzas Armadas (la Reserva Voluntaria, puesta en marcha en 2003 y mediante la cual se crea la figura del llamado "militar a tiempo parcial", consistente en la incorporación temporal a las Fuerzas Armadas de civiles con las Categorías de Oficial, Suboficial o Tropa y Marinería para el desempeño de sus profesiones u oficios) la presencia de mujeres es notablemente más elevada que en el ámbito profesional militar. En concreto, desde su puesta en marcha, las cifras que arrojan son que uno de cada cuatro reservistas son mujeres. El hecho de que en tan poco tiempo de vigencia de la Reserva Voluntaria la presencia femenina sea de tal entidad nos permite ser optimistas con respecto al interés profesional (aunque sea de manera temporal) que las Fuerzas Armadas despiertan entre las mujeres y a que la participación en la Defensa Nacional sea visto como una responsabilidad compartida entre ambos sexos. La figura del Reservista es clave en la cultura de la Defensa.

Dentro de las Fuerzas Armadas, las mujeres pilotan aeronaves en sus distintas facetas (caza, transporte, hidroaviones y helicópteros), son expertas en guerra electrónica, conducen carros de combate, prestan servicio como comandante de patrullera y en las dotaciones de los submarinos, pertenecen a unidades de élite como la Brigada Legionaria y la Brigada Paracaidista y desempeñan profesiones técnicas, jurídicas, económicas y sanitarias en el ámbito de la Defensa. Y todo ello lo realizan tanto dentro como fuera de nuestras fronteras, integradas en los contingentes desplegados en las diferentes Misiones Internacionales en las que nuestro país participa. Las mujeres militares, al igual que el conjunto de los miembros de las Fuerzas Armadas, están participando en la consecución de las misiones, aportando su experiencia, su esfuerzo y asumiendo los riesgos inherentes a esta profesión. 
Nadie puede dudar del máximo compromiso y entrega de las mujeres en esta profesión.

Sin embargo, la presencia de la mujer en las Fuerzas Armadas no se reduce a un mero incremento de efectivos, sino que ha conllevado y conlleva un preceptivo e ingente cambio normativo, cultural, logístico, de infraestructuras y de usos y costumbres. No olvidemos que ha sido toda una organización militar tradicionalmente integrada por hombres, la que ha tenido que adaptarse a su nueva realidad.

Efectivamente, la integración se define como "un proceso bidireccional y dinámico de ajuste mutuo por parte de todos los implicados". Esta formulación encierra dos ideas clave: en primer lugar, que la integración, más que un estado de cosas en un momento determinado, es un proceso continuo, prolongado en el tiempo, que tiene que ser permanentemente reproducido y renovado; en segundo lugar, que la integración requiere un esfuerzo mutuo o bidireccional de adaptación a la nueva realidad tanto por parte de las mujeres como de los varones que viven este proceso. Por lo tanto, hay también que felicitarles, en estos momentos, a todos ellos.

Un proceso de estas características, además de la colaboración y cooperación de todos los que integran las Fuerzas Armadas, requiere, para llegar a buen puerto, un decidido impulso desde los órganos superiores de la Defensa tanto para remover los obstáculos que pudieran impedir que la integración sea un hecho como para facilitar el cumplimento de los deberes militares al conjunto de sus miembros, independientemente de su sexo, pero con especial énfasis en las mujeres debido a su incorporación posterior secularmente al varón. Por ello, el Ministerio de Defensa ha implantado medidas necesarias tendentes a la plasmación del principio de igualdad de oportunidades, en cuanto al acceso, permanencia y carrera profesional de las mujeres. En este sentido podemos citar como la propia Ley de carrera militar, en cuyo artículo 6 establece la igualdad entre las mujeres y hombres como principio transversal, al regular en su párrafo primero que:

"La igualdad de trato y oportunidades en un principio que en las Fuerzas Armadas se aplicará de conformidad con lo previsto en la Ley Orgánica 3/2007, de 22 de marzo, para la igualdad efectiva de mujeres y hombres y que estará especialmente presente en el desarrollo y aplicación de esta ley en lo relacionado con el acceso, la formación y la carrera militar".

Tal y como se ha dicho, se han adoptado medidas como la creación del Observatorio de Mujer en las Fuerzas Armadas, el desarrollo del Programa de Es- cuelas Infantiles (que ha servido para poner en funcionamiento, en colaboración con las CC.AA. este tipo de centros de enseñanza en establecimientos militares para facilitar la conciliación de la vida personal y profesional de los hombres y las mujeres que prestan servicio en nuestras Fuerzas Armadas), la adaptación de infraestructuras y de la uniformidad, la ejecución de actuaciones específicas dirigidas a la mujer en el ámbito de la salud o de la conciliación, la participación de las mujeres, en la medida que sea posible, en las Juntas de evaluación y ascenso, o la aplicación del criterio de género en las Reales Ordenanzas de las Fuerzas Armadas.

En cuanto al Observatorio de la Mujer en las FAS, se señala que era un órgano colegiado de asesoramiento dependiente de la Subsecretaría de Defensa, cuya finalidad fundamental es analizar y promover la integración y permanencia de la mujer en las Fuerzas Armadas, así como para asesorar en materia de igualdad y conciliación. Mediante la Orden Ministerial 51/2011, de 28 de julio, se cambia el nombre y la organización del Observatorio de la Mujer en las FAS pasándose a denominar el Observatorio Militar para la igualdad entre mujeres y hombres en las Fuerzas Armadas.

El Observatorio Militar para la igualdad es el órgano encargado de velar la consecución de la igualdad real y efectiva entre mujeres y hombres en las Fuerzas Armadas y el fomento de medidas de conciliación de la vida personal familiar y profesional. Este Observatorio es un órgano colegiado de carácter consultivo adscrito a la Subsecretaría de Defensa, a través de la Dirección General de Personal.

Tras la aprobación de la Orden Ministerial 51/2011, de 28 de julio, el pasado 3 de diciembre se celebró el Pleno constitutivo del Observatorio Militar para la Igualdad, en el que se aprobó el reglamento interno de funcionamiento, y se constituyeron las comisiones técnicas de funcionamiento. El nuevo Observatorio Militar para la Igualdad entre mujeres y hombres en las Fuerzas Armadas ha permitido no solo un cambio de denominación, estructura y funcionamiento sino, una mejora de la eficacia y eficiencia en su actuación. Además, se ha impulsado la Secretaría Permanente de Igualdad, como órgano de apoyo técnico y administrativo al Observatorio Militar para la Igualdad en los periodos en los que este no está reunido, dando continuidad a su actuación. La Secretaria asume además las funciones propias de las unidades de igualdad en los términos previstos por el artículo 77 de la Ley Orgánica 3/2007, de 22 de marzo, para la igualdad efectiva de mujeres y hombres. 
Asimismo, este Observatorio cuenta con una página web, un correo electrónico y un teléfono de información. Esta comunicación permite, por un lado, encauzar las inquietudes y sugerencias sobre igualdad y conciliación, y, por otro, conocer aspectos mejorables en este ámbito, así como ser un referente al respecto en el seno de las Fuerzas Armadas españolas.

Por otra parte, me gustaría hacer mención especialmente a las Reales Ordenanzas aprobadas por el Real Decreto 96/2009, de 6 de febrero, en tanto que recogen y definen los principios éticos y las reglas de comportamiento del militar de acuerdo con la Constitución, la Ley Orgánica 5/2005, de 17 de noviembre, de la Defensa Nacional y la Ley de la carrera militar. Deben servir de guía a todos los militares para fomentar el exacto cumplimiento del deber, inspirado en el amor a España y en el honor, disciplina y valor.

Precisamente, y en este contexto, es dónde es especialmente importante analizar las mismas en clave de género. Las Reales Ordenanzas persiguen, entre otros objetivos, que la igualdad efectiva entre hombres y mujeres y todo lo que ello deriva se configure como un principio informador básico de la actuación del personal militar. Es la primera vez que en las reglas que rigen el comportamiento del personal militar se introducen estos principios. Asimismo, se ha tenido presente y se ha regulado no solamente desde la perspectiva del comportamiento y actuación entre los y las militares, sino en sus relaciones con la sociedad civil, y especialmente en el escenario de las Operaciones, introduciendo los mandatos Internacionales al respecto.

\section{APORTACIÓN DE LA MUJER MILITAR EN LAS OPERACIONES, EN EL CONTEXTO DE LAS RESOLUCIONES DE NACIONES UNIDAS SOBRE MUJER, PAZ Y SEGURIDAD}

El punto de partida es la Resolución 1325 sobre mujer, paz y seguridad, aprobada por el Consejo de Seguridad de Naciones Unidas en su sesión 4213a, celebrada el 31 de octubre de 2000. La citada Resolución ${ }^{7}$ insta a los Estados y al Secretario General de Naciones Unidas a aplicar el criterio de género en las situaciones de conflictos armados y reconstrucción de paz, respecto a personal desplegado (militar o civil) y población local.

Esta Resolución constituye el marco político decisivo para incorporar la perspectiva de género en la prevención, gestión y solución de los conflictos armados y se ha erigido como punto de partida y principio informador de actuación para todos los actores invo- lucrados o afectados en estos escenarios. Es importante, también, desde un punto de vista operativo y de eficiencia.

El año 2010 se cumplieron 10 años desde su aprobación, y por ello fue objeto de análisis, evaluación e impulso desde diferentes ámbitos, culminando con la adopción de la Resolución 1960, de diciembre de 2010, del Consejo de Seguridad de Naciones Unidas, en la que se refuerza el contenido de la 1325 mencionada.

En estos últimos años se ha trabajado en su puesta práctica desde ámbitos nacionales, gubernamentales y no gubernamentales, Internacionales y supranacionales. (Estados, NGOs, Naciones Unidas ${ }^{8}$, OTAN, UE).

En este sentido, se ha recomendado que los Estados adopten planes nacionales de acción concretos, como una herramienta de actuación eficaz al respecto.

Estos planes deben contener actuaciones tales como; La formación en cuestiones de género al personal desplegado, la adopción de políticas de igualdad en las Fuerzas Armadas, el conocimiento de la situación de la mujer y la infancia en los escenarios de las operaciones, el acercamiento y apoyo a la mujer local, la recopilación estadística y de datos e informes de una operación desagregada por sexo, la introducción del criterio de género en las evaluaciones de las misiones, o la creación de asesores de género en las operaciones.

La cumbre de OTAN de Chicago celebrada los días 20 y 21 de mayo de 2012, ha significado un apoyo decisivo a la aplicación de las resoluciones sobre mujeres, paz y seguridad que nacen con la Resolución $1325^{\circ}$. Así, entre las conclusiones aprobadas, destaca el compromiso de los Aliados y de la Organización a la completa implementación de las Resoluciones de Naciones Unidas sobre mujeres, paz y seguridad y reconocen el trabajo realizado por la Alianza, que en la actualidad cuenta con una Representante especial para estos fines.

Sólo $17^{10}$ países han adoptado planes de acción nacionales, entre ellos España que cuenta con un Plan de Acción aprobado mediante Acuerdo de Consejo de Ministros de noviembre de 2007. En su elaboración participaron, bajo la coordinación de la Embajadora en Misión especial para las cuestiones de género del Ministerio de Asuntos Exteriores y Cooperación, distintos Departamentos ministeriales, entre ellos, el Ministerio de Defensa.

Por otra parte, algunas organizaciones internacionales regionales y sub-regionales han optado también 
por desarrollar Planes de Acción Regionales para la implementación de la Resolución 1325 y Resoluciones conexas, ya sea como parte de una política general en materia de género o como estrategias separadas. Como ejemplos pueden citarse los planes de acción de la OTAN, de la Unión Europea, la OSCE y la Unión Africana, entre otros.

En cuanto al contenido del Plan español, se señala que éste se ha articulado en torno a seis objetivos, siguiendo el esquema de la Resolución 1325:

1. Potenciar la participación de mujeres en las misiones de paz y en sus órganos de toma de decisiones.

2. Promover la inclusión de la perspectiva de género en todas las actividades de construcción de la paz.

3. Asegurar la formación específica del personal que participa en operaciones de paz, en materia de igualdad y sobre los distintos aspectos de la Resolución 1325, además de fomentar el conocimiento y difusión de la misma.

4. Proteger los derechos humanos de las mujeres y niñas en zonas de conflicto y posconflicto (incluyendo campos de personas refugiadas y desplazadas) y fomentar el empoderamiento y la participación de las mujeres en los procesos de negociación y aplicación de los acuerdos de paz.

5. Incorporar el principio de igualdad de trato y oportunidades entre mujeres y hombres en la planificación y ejecución de actividades para el Desarme, la Desmovilización y la Reintegración (DDR), así como el adiestramiento especializado al respecto de todo el personal que participa en dichos procesos.

6. Fomentar la participación de la sociedad civil española en relación con la Resolución 1325.

Todos estos objetivos parten de la idea de que a la hora de poner en marcha procesos relacionados con la seguridad y la construcción de alternativas viables de paz en zonas de conflicto y posconflicto, no se pueden obviar el enfoque de género, ni las situaciones y necesidades específicas de las mujeres y de las niñas.

Todas estas actuaciones parten de la constatación de que las mujeres y los hombres se complementan en sus capacidades y son esenciales para la efectividad de las Misiones, sobre todo en el contexto de la Seguridad actual. La aplicación del criterio de género es un elemento clave para la completa efectividad de las operaciones actuales.
Precisamente, y en este contexto, es necesario finalizar esta breve exposición recordando a la Soldado de Infantería Ligera Idoia Rodríguez Bujan, primera mujer militar fallecida mientras cumplía con su deber en Afganistán, formando parte del contingente español desplegado en dicho país en el marco de la Operación ISAF. Su muerte el 21 de febrero de 2007 mientras cumplía con sus obligaciones como militar tiene un especial significado en el proceso de incorporación de la mujer en nuestras Fuerzas Armadas. Su desaparición ha dejado patente que en la labor que hombres y mujeres llevan a cabo cada día en el seno de nuestros Ejércitos, no existen diferencias en su responsabilidad y compromiso. Las misiones, funciones y riesgos que se asumen afectan a todos por igual sin que se pueda hablar de distinción por razón de sexo, y los tradicionales valores que definen el espíritu militar, como patriotismo, vocación, disciplina, entrega y sacrificio, atañen por igual a los hombres y mujeres que forman parte de nuestras Fuerzas Armadas y son asumidos por todos de igual manera.

En su memoria el Ministerio de Defensa creó el Premio Soldado Idoia Rodríguez para reconocer la labor de aquellas personas físicas o jurídicas, colectivos o instituciones, tanto militares como civiles, que hubiesen realizado actuaciones relevantes o ejemplares para potenciar el papel de la mujer o para apoyar la igualdad de oportunidades y de género en las Fuerzas Armadas.

La primera edición de este Premio (año 2008) recayó sobre las mujeres que se incorporaron a las Fuerzas Armadas, en las primeras promociones correspondientes de sus diferentes Cuerpos y Escalas, como reconocimiento a su labor colectiva al abrir el camino, desde un punto de vista profesional y social, a que muchas otras mujeres hayan ingresado con posterioridad a nuestros Ejércitos. La segunda Edición del mismo (año 2009), recayó el galardón en la Cabo del Ejército de Tierra Doña Lucía Peraita García en reconocimiento a su excelente trayectoria profesional y a sus virtudes militares y personales acreditadas en el desarrollo de sus cometidos en las Misiones Internacionales realizadas así como en sus diferentes destinos, según se recoge en la Orden DEF/1592/2009, de 10 de junio, por la que se concede el premio «Soldado Idoia Rodríguez, Mujer en las Fuerzas Armadas, para el año 2009. En la tercera Edición (2010) se premió a Dạ Valentina Fernández Vargas, investigadora del CSIC, por su larga trayectoria dedicada a la investigación y difusión de cuestiones de género y Fuerzas 
Armadas, y su apoyo académico a la igualdad y los derechos de la mujer militar en España, según se recoge en la Orden DEF/2723/2010, de 13 de octubre. En la última edición de este Galardón, el Premio recayó en el Comité de Perspectiva de Género de las Fuerzas Armadas OTAN, según la Orden DEF/3513/2011, de 30 de noviembre de 2011, en reconocimiento a su apoyo a la incorporación e integración de la mujer en las Fuerzas Armadas españolas y países aliados, y por su trabajo para la aplicación, en el seno de la Alianza de la perspectiva de género y de las Resoluciones del Consejo de Seguridad de las Naciones Unidas sobre mujeres, paz y seguridad.

\section{CONCLUSIONES}

Por todo lo expuesto, no hay duda que mucho se ha conseguido en 25 años, alcanzando metas muy ambiciosas. No obstante, se debe seguir trabajando para que la igualdad efectiva entre mujeres y hombres militares sea una realidad completa, con el objetivo de hacer posible que la carrera militar no sea más gravosa para la mujer por el simple hecho de serlo.

La contribución de las mujeres de forma activa y profesional a la Defensa y Seguridad de nuestro país se ha consolidado como una cuestión de derecho, de eficacia, eficiencia y operatividad, redundando en la seguridad de todos.
1 El artículo 2 de la derogada Ley Orgánica 6/1980, de 1 de julio, por la que se regulan los criterios básicos de la Defensa Nacional y la Organización Militar establecía que la finalidad de la Defensa era garantizar de modo permanente la unidad, soberanía e independencia de España, su integridad territorial y el ordenamiento constitucional, protegiendo la vida de la población y los intereses de la patria, en el marco de lo dispuesto en el artículo 97 de la Constitución.

2 La Ley Orgánica de Defensa Nacional $5 / 2005$, de 17 de noviembre, establece que la política de defensa tiene por finalidad la protección del conjunto de la sociedad española, de su Constitución, de los valores superiores, principios e instituciones que en ésta se consagran, del Estado social y democrático de derecho, del pleno ejercicio de los derechos y libertades, y de la garantía, independencia e integridad territoria de España. Asimismo, tiene por objetivo contribuir a la preservación de la paz y seguridad internacionales, en el marco de los compromisos contraídos por el Reino de España.

3 Real Decreto-ley 1/1988, de 22 de febrero, se regula, por primera vez, la incorporación de la Mujer a las Fuerzas Armadas.

4 Real Decreto 984/1992, de 31 de julio por el que aprueba el Reglamento de Tropa y Marinería Profesionales en las Fuerzas Armadas.
5 Ley 17/1999, de 18 de mayo, de Régimen del Personal de las Fuerzas Armadas.

6 Ley 39/2007, de 19 de noviembre, de Carrera Militar.

7 Hace mención a la necesidad de tener en cuenta este criterio en el planeamiento, ejecución y evaluación de una Misión, establece la necesidad de incrementar la participación de las mujeres, en condiciones de igualdad, en los procesos de toma de decisión que tengan lugar para el fomento de la paz y la seguridad, así como en la prevención y solución de conflictos y en las operaciones de mantenimiento de la paz. Otro aspecto que identifica es la necesidad de asegurar la formación específica del personal que participa en las operaciones, para garantizar a las mujeres y niñas en zonas de conflicto y posconflicto el pleno disfrute de los derechos humanos.

8 Dentro de Naciones Unidas se han sucedido diversas actuaciones operativas concretas, y se han aprobado otras Resoluciones que profundizan determinados aspectos relacionados con la citada RCSNU 1325 como las Resoluciones 1820 (2008), 1888 (2009) y 1889 (2009), 1960 (2010) todas ellas sobre mujeres, paz y seguridad.

9 16. Widespread sexual and genderbased violence in conflict situations, the lack of effective institutional arrangements to protect women, and the continued under-representation of women in peace processes, remain serious impediments to building sustainable peace. We remain committed to the full implementation of United Nations Security Council Resolution (UNSCR) 1325 on Women, Peace and Security and related Resolutions which are aimed at protecting and promoting women's rights, role, and participation in preventing and ending conflict. In line with the NATO/ Euro-Atlantic Partnership Council (EAPC) Policy, the Alliance, together with its partners, has made significant progress in implementing the goals articulated in these Resolutions. In this regard, we have today endorsed a Strategic Progress Report on mainstreaming UNSCR 1325 and related Resolutions into NATO-led Operations and Missions, and welcomed Norway's generous offer to provide a NATO Special Representative for these important issues. In this context, and to further advance this work, we have tasked the Council to: continue implementing the Policy and the Action Plan; undertake a review of the practical implications of UNSCR 1325 for the conduct of NATO operations and missions; further integrate gender perspectives into Alliance activities; and submit a report for our next Summit.

10 Austria, Bélgica, Chile, Costa de Marfil, Dinamarca, Finlandia, Islandia, Liberia, Países Bajos, Noruega, Portugal, Sierra Leona, Suecia, Suiza, Uganda, Reino Unido, y España. 